\title{
Rhino cerebral mucormycosis. A report of two cases and review of literature.
}

\author{
Reddy S. Sujatha ${ }^{1}$, N. Rakesh ${ }^{2}$, Jatti Deepa ${ }^{3}$, Lanjekar Ashish ${ }^{3}$, Bijjal Shridevi ${ }^{4}$ \\ ${ }^{1}$ Professor. Dept of Oral Medicine, Diagnosis and Radiology. M.S.Ramaiah Dental College \& Hospital, Msrit Post, New Bel \\ Road, Bangalore-560054. Karnataka (India) \\ ${ }^{2}$ Senior Lecturer. Dept of Oral Medicine, Diagnosis and Radiology. M.S.Ramaiah Dental College \& Hospital, Msrit Post, New \\ Bel Road, Bangalore-560054. Karnataka (India) \\ ${ }^{3}$ PG Students. Dept of Oral Medicine, Diagnosis and Radiology. M.S.Ramaiah Dental College \& Hospital, Msrit Post, New Bel \\ Road, Bangalore-560054. Karnataka (India) \\ ${ }^{4}$ Senior lecturer. Dep of Oral Medicine and Radiology.Bhartiya Vidyapeeth Dental College. Sangli, Maharashtra.
}

Correspondence:

Dept of Oral Medicine, Diagnosis and Radiology.

M.S.Ramaiah Dental College \& Hospital,

Msrit Post, New Bel Road,

Bangalore-560054.

Karnataka (India).

E-mail id: s_sujathajanardhan@yahoo.com

Sujatha RS., Rakesh N.,Deepa J., Ashish L., Shridevi B. Rhino cerebral mucormycosis. A report of two cases and review of literature. J Clin Exp Dent. 2011;3(3):e256-60.

http://www.medicinaoral.com/odo/volumenes/v3i3/jcedv3i3p256.pdf

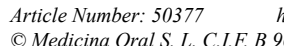

\begin{abstract}
Mucormycosis is caused by fungi of the order Mucorales and is one of the fulminant and fatal mycotic infections known to human beings with a high mortality rate. Rhinocerebral mucormycosis is the most common type and its extension to the orbit and brain is quite usual. It is commonly reported in immunocompromised patients such as poorly controlled diabetes mellitus, blood dyscrasias, malnutrition, neutropenia, iron overload, organ transplant, and immunosuppressive therapy. Mucormycosis is on the rise with an increase in incidence of Diabetes mellitus and HIV infection leading to immunocompromised status of the patient. Here in, reporting two cases of rhinocerebral mucormycosis in two uncontrolled diabetic patients with deep palatal perforation in one case and this is a rare and late occurrence. Both the patients were successfully treated with a combination of surgical debridement and systemic amphotericin B administration. By presenting this case report we would like to point out that mucormycosis should be included in the differential diagnosis of hard palate ulcers and cellulitis.
\end{abstract}

Key words: Fungal infection, mucorales, immunocompromised status, palatal perforation, diabetes mellitus. 


\section{Introduction}

Mucormycosis (Zygomycosis) is a rare, opportunistic fungal infection, occasionally fatal caused by mucorales, belonging to the class of zygomycetes (phycomycetes) (1) This fungus is widespread and occurs in soil, manure, vegetable, fruits and as bread mold. The incidence of mucormycosis is approximately 1.7 cases per $1,000,000$ inhabitants per year.(2) Depending on the immunological status of the patient, the disease may manifest in six different ways depending on the affected site as rhinocerebral, pulmonary, cutaneous, gastrointestinal, central nervous system or disseminated forms. Patients with diabetes mellitus usually have the rhinocerebral and pulmonary forms of the disease (3). Diagnosis is confirmed by histopathological demonstration of the organism in the affected tissue (4). Early diagnosis and treatment of mucormycosis is extremely important due to the aggressive course of the disease. Control of underlying disease must be established, metabolic abnormalities corrected and antifungal therapy should be combined with surgical debridement of all necrotic tissues (5).

Herein, reporting two cases of rhino cerebral mucormycosis which were treated by a combined approach involving, medical and surgical management.

\section{Case Report}

\section{Case Report 1}

A 65 year old female patient reported to the dental department with a complaint of nasal regurgitation of food associated with a purulent discharge from the nasal cavity and right eye for the past 15 days (Fig .1). Patient noticed drooping of her upper right eyelid and complete closure of her right eye associated with fever and pain

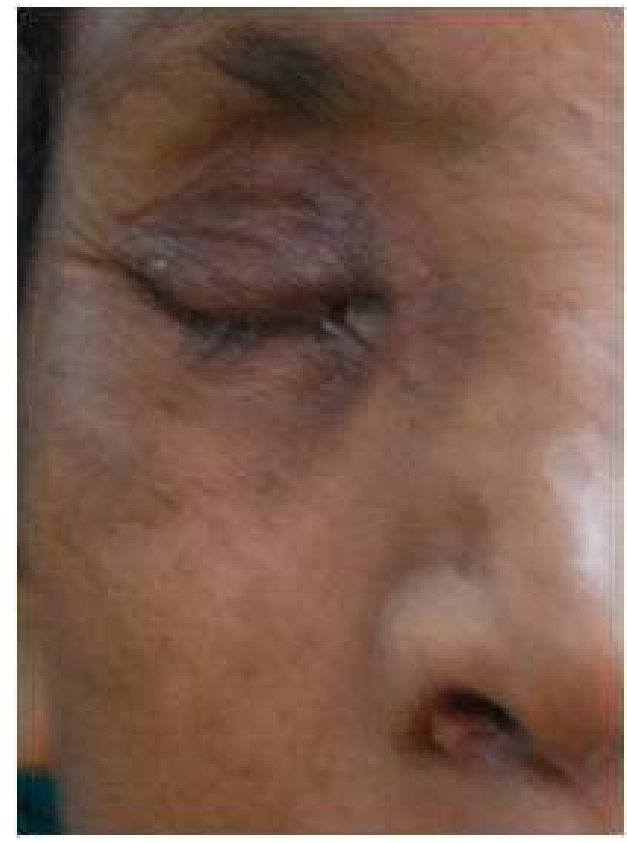

Fig. 1.Complete drooping of upper eyelid with pus discharge

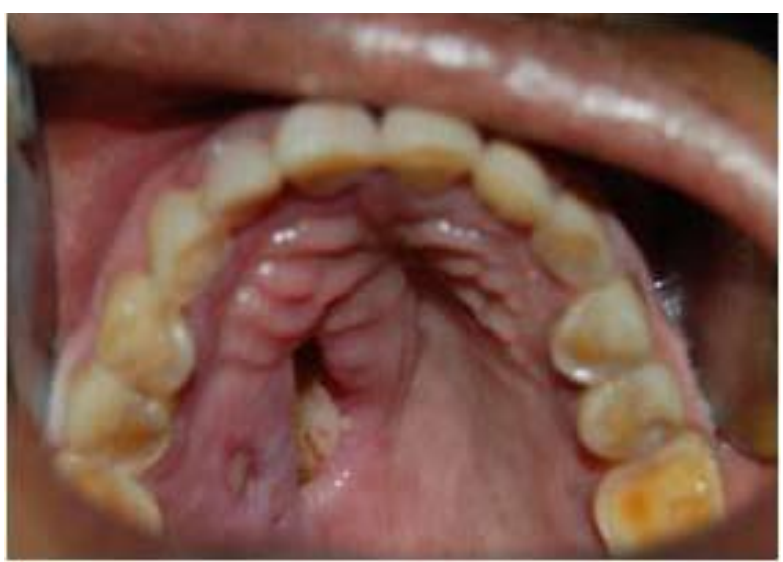

Fig 2. Perforation in the right anterior region of the hard palate

on the right side of the face (Fig.1). Patient is a known diabetic and hypertensive since 10 years with a history of poor drug compliance. Patient was poorly built and debilitated, confused, disoriented and foul smelling. There was ptosis; complete drooping of her right upper eyelid with purulent discharge and inability to separate the eyelids. Based on these findings a diagnosis of right eye panopthalmitis was given. Examination of the nasal cavity revealed eschar of the right nasal septum with exposure of bone. Intraorally $3 \times 2 \mathrm{~cm}$ circular perforation was noticed in the anterior region of the hard palate through which a blackish, necrotic hemorrhagic mass was seen (Fig.2). A through and through opening was formed due to perforation in the palate creating an oronasal fistula. Submandibular lymphadenopathy was present. Based on the history and clinical presentation, a provisional diagnosis of mucormycosis was made and gummatous necrosis, midline lethal granuloma and Wegener's granulomatosis were considered under differential diagnosis. Patient was subjected to various biochemical investigations (Table 1).

\begin{tabular}{|l|l|}
\hline Random blood sugar & $419 \mathrm{mg} / \mathrm{dl}$ \\
\hline Hemoglobin & $9.6 \mathrm{gm} \%$ \\
\hline Packed cell volume & $32 \%$ \\
\hline Ketone bodies & Negative \\
\hline CSF examination & \\
Glucose & $155 \mathrm{mg} \%$ \\
Protein & $53 \mathrm{mg} \%$ \\
Chlorine & $127 \mathrm{meq} / 1$ \\
\hline HIV & negative \\
\hline
\end{tabular}

CSF - Cerebrospinal Fluid

HIV-Human Immunodeficiency Virus

Table 1. Biochemical investigations of case 1 


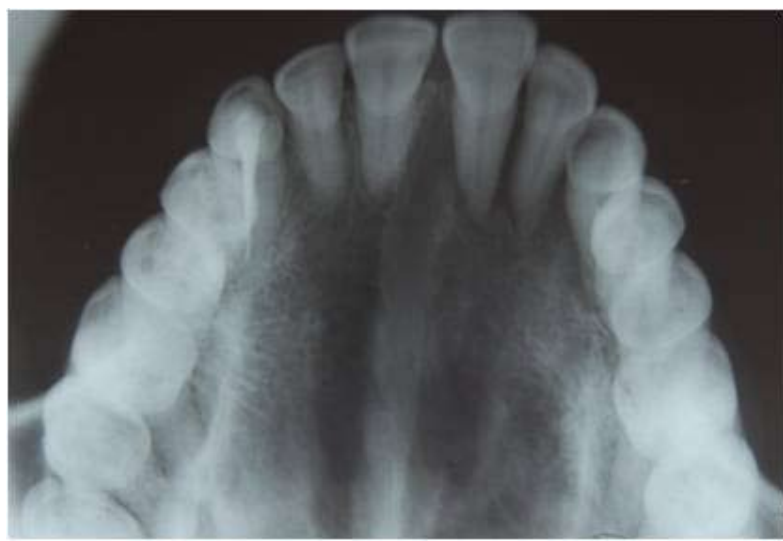

Fig. 3. Occlusal radiograph showing ill-defined radiolucency in the anterior region of the hard palate

Occlusal radiograph showed an ill-defined radiolucency in the anterior region of the hard palate (Fig.3). Water's view showed diffuse haziness of the right maxillary sinus with evidence of destruction of floor of the sinus. Magnetic resonance imaging (MRI) did not show any significant changes. Scrapings of conjunctiva, nasal cavity and palate were sent for histopathological examination. The histopathological report revealed nonseptate hyphae branched at right angle in areas of exudate and granulation tissue, a feature of deep fungal infection.

Surgical, Ear Nose Throat (ENT), neurological and histopathologic report concurred with the diagnosis of rhino cerebral mucormycosis. Emergency medical management of diabetes was started by administration of insulin which resulted in optimal glycaemic control. The patient was started on intravenous amphotericin B $50 \mathrm{mg}$ in 3 divided doses and metronidazole $1 \mathrm{gm}$ intravenously every 12 hourly. Patient was transfused with 1 unit of packed cells after blood grouping and cross matching. Evisceration procedure of the right eye was performed after monitoring blood glucose levels and other vital signs. Surgical debridement of necrotic tissues of nasal wall and palate was done following which an obturator was constructed for the patient. Thus, with a combined approach of dental, ophthalmological, surgical and ENT team the case was successfully treated. The patient was advised daily administration of insulin and to maintain glycaemic control, scrupulous oral and general hygiene. Patient is under follow up.

\section{Case Report 2}

A 57 year old male patient, farmer by profession reported with a complaint of pain in the left upper and lower jaw since a month and swelling on the left side of the face since three weeks. Pain was continuous, throbbing in nature, radiating to the head and neck region, which was relieved on taking over the counter analgesics. Intermittent blood- tinged nasal discharge since 1 week was reported. Patient was a known diabetic since 7 years and was on insulin therapy 40 IU once a day. He was

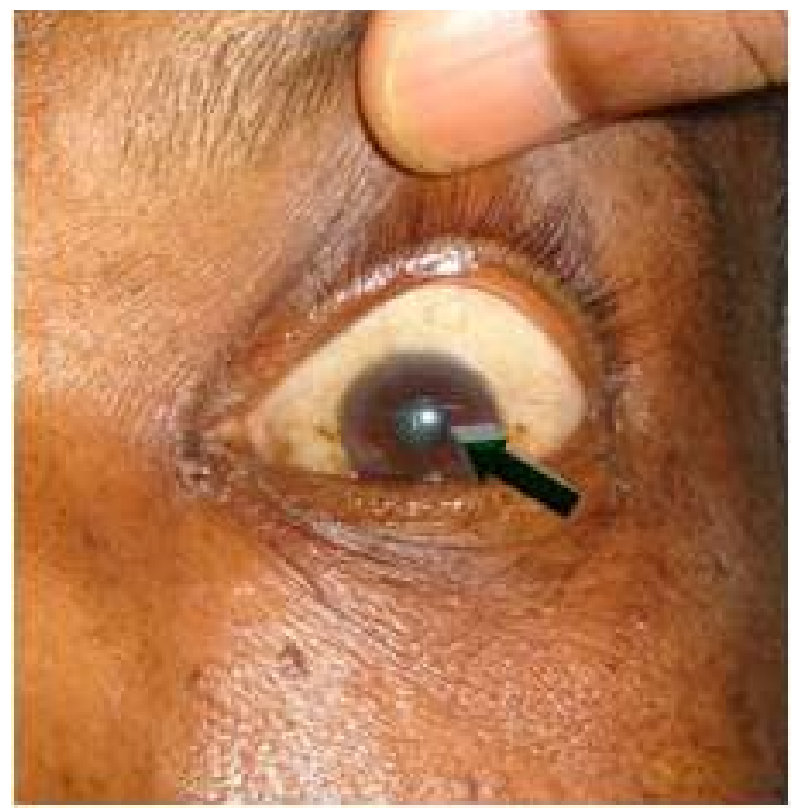

Fig. 4. Dilated pupil of the left eyeeye

moderately built and nourished and vital signs were within the normal range.

Extraoral examination revealed a diffuse swelling on the left half of the face with periorbital edema. On palpation the swelling was tender, febrile and soft in consistency. Submandibular lymphadenopathy was noticed. Examination of left eye revealed diminished vision, ptosis, proptosis, dilated pupil, and restricted eye movement suggestive of involvement of 2nd, 3rd, 4th and 6th cranial nerves (Fig. 4).Intraoral examination revealed bad oral hygiene, generalized gingival recession, attrition, and cervical abrasion. Based on history and clinical examination, a working diagnosis of cellulitis was given and canine space infection and cavernous sinus thrombosis were considered under differential diagnosis. Blood sugar and other investigations were carried out (Table 2).

\begin{tabular}{|l|l|}
\hline Random blood sugar & $392 \mathrm{mg} / \mathrm{dl}$ \\
\hline Hemoglobin & $12 \mathrm{gm} \%$ \\
\hline RBC & $4.6 \mathrm{millions} / \mathrm{mm} 3$ \\
\hline WBC & $7,000 / \mathrm{ML}$ \\
\hline Differential count & 62 \\
Neutrophils Lymphocytes & 23 \\
Eosinophils & 2 \\
Basophils & 0 \\
Monocytes & 3 \\
\hline HIV & negative \\
\hline
\end{tabular}

WBC-White Blood Cells

HIV-Human Immunodeficiency Virus

Table 2. Biochemical investigations of case 2 

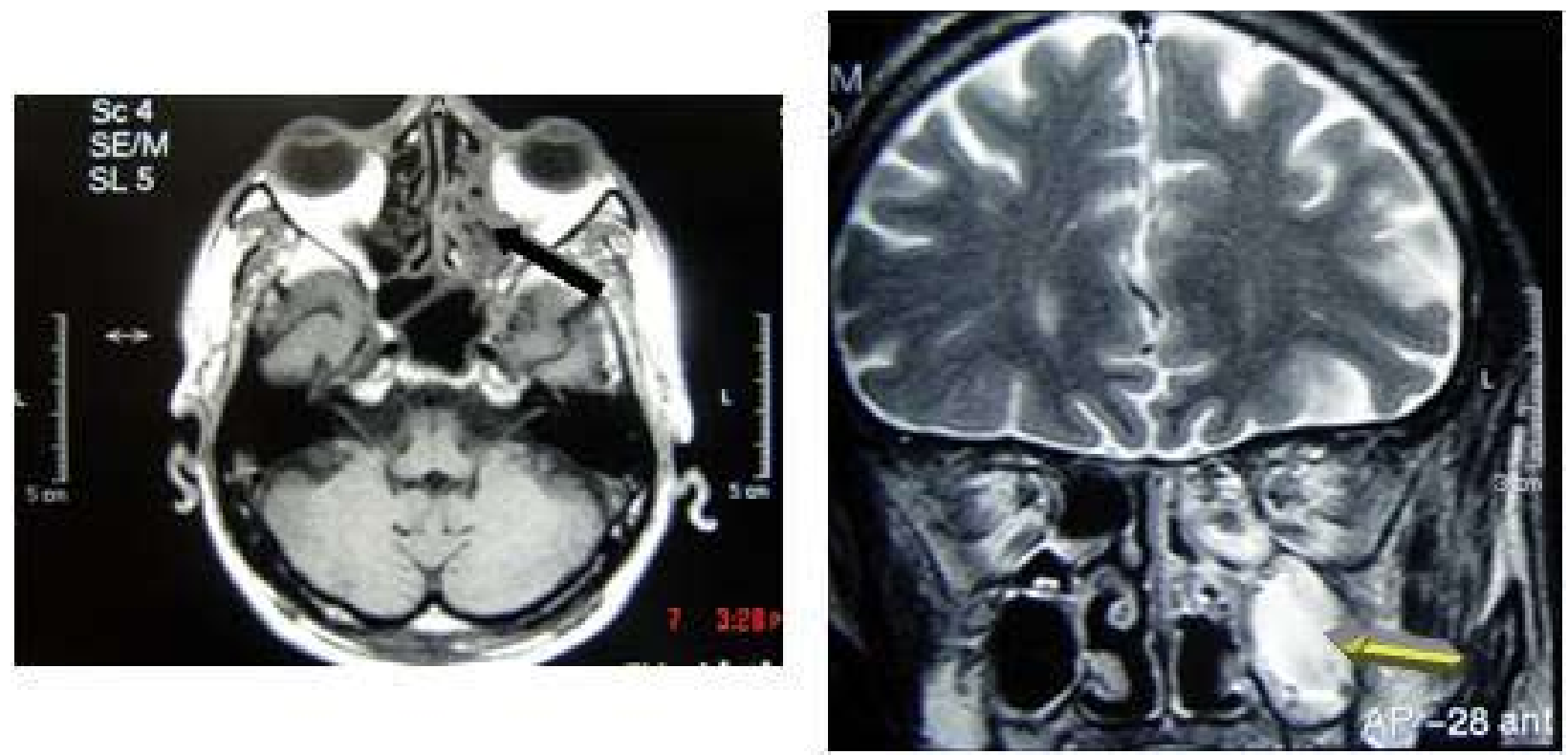

Fig. 5. T2 weighted image shows hyperintense region in ethmoid sinus and maxillary sinus

Paranasal sinus view revealed haziness on left maxillary sinus region. MRI suggested ethmoid, frontal, and maxillary sinus opacification (Fig.5).Patient was referred to department of ophthalmology for eye examination and department of otolaryngology for further evaluation where functional endoscopic sinus surgery was performed and tissue specimen was sent for the histopathologic examination. It revealed broad non-septate fungal filaments with branching at right angles. Based on the above findings a final diagnosis of the rhino orbital-cerebral mucormycosis was given.

Human mixtard $40 \mathrm{IU} / \mathrm{ml}$ and Human actraped $40 \mathrm{IU} / \mathrm{ml}$ was administered till blood glucose levels were within the normal range. Debridement of sinus was done and parenteral Ceftriaxone 1gm, amikacin $500 \mathrm{mg}$ and amphotericin B $50 \mathrm{mg}$ diluted in $500 \mathrm{ml}$ normal saline per day was prescribed for 7 days and injection amphotericin B $50 \mathrm{mg}$ once a day for 3 months was advised. Nasal wash with saline was advised for a week. His condition improved dramatically and is under follow up.

\section{Discussion}

Mucormycosis infection in humans is usually acquired through airborne fungal spores, contamination of traumatized tissue, ingestion and direct inoculation (6).For optimal growth in humans, mucorales fungi require the host to have decreased levels of neutrophils, since they play a key role in defense against the fungi. In healthy individuals neutrophils phagocytose these pathogens whereas in neutropenic individuals such as in chemotherapy patients or malignancy, pathogens proliferate in the host tissue and infection ensues (7).

While mucormycosis has been reported in otherwise healthy individuals; a predisposing medical condition is virtually always present. Both our patients had uncontrolled diabetes which is a well-known predisposing factor for mucormycosis. Uncontrolled diabetes mellitus can alter the normal immunologic response of patients to infections. The number of spores also rapidly increase in carbohydrate-rich environment .Such patients have decreased granulocyte phagocytic ability with altered polymorphonuclear leukocyte response (8).Diabetes mellitus is most common and is associated with $40 \%$ of mucormycosis cases overall and $70 \%$ of patients with rhino cerebral mucormycosis (7).

Rhino cerebral mucormycosis usually begins in the nasal mucosa or palate and extends to the paranasal sinuses, spreading via the angular, lacrimal, and ethmoid vessels as well as by direct extension from the sinuses into the retro-orbital region. Once fungal hyphae enter into the blood stream they can disseminate to other organs such as cerebrum or lungs which can be fatal for the patient. Mucor hyphae form thrombi within the blood vessels which reduce vascularity to the tissues and cause necrosis (9). Thrombosis of the internal maxillary artery or sphenoplatine artery caused by mucormycotic infection as well as immunocompromised state due to chronic uncontrolled diabetes in our second patient may have resulted in palatal perforation and necrosis of the right nasal septum. With orbital involvement, function of the cranial nerves II, III, IV and VI may be lost or impaired, with resultant proptosis, ptosis, papillary dilation, visual loss as well as periorbital cellulitis. Typical clinical presentation includes facial pain, presence of an irregular black eschar on the palatal or nasal mucosa and drainage of pus from the eye and nose (10).All these findings are consistent with both our cases and are suggestive of rhino cerebral mucormycosis.

Radiographically, rhinocerebral mucormycosis has been 
associated with nodular thickening of sinus mucosa, sinus opacification without fluid levels, and spotty destruction of the bony walls of paranasal sinuses. It has been suggested that any diabetic patient in a ketoacidotic state who presents with clinical and radiographic findings of rhino sinusitis should be suspected as having mucormycosis until proven otherwise (11).

Early diagnosis and treatment are of extreme importance for successful eradication of infection and for patient survival. For a definitive diagnosis of mucormycosis, the histopathological specimen need not be of the isolated fungus, but may be a sample of the necrotic tissue from the site in question. The Grocott-Gomori methenamine silver stain is the most effective for identifying fungi. However, haemotoxylin and eosin, periodic acid schiff or calcofluor white stains may also be used. Invasion of the tissue by fungal non septate hyphae and right angle branching is present in mucormycosis specimens. Because initial cultures of diseased tissue may be negative, histopathological examination is essential for early diagnosis $(10,12)$.

Systemic antifungal therapy includes the use of high dose amphotericin-B and is associated with an overall survival rate of $72 \%$. Since its usage is associated with renal toxicity and it requires careful monitoring of serum urea nitrogen, blood urea nitrogen potassium, creatinine as well as creatinine clearance as an essential part of the therapy. Recently, studies have shown that liposomal amphotericin B (where the drug has been inserted into liposomes) (13) and fluconazole (14) can be used with good success. Hyperbaric oxygen therapy is believed to improve neutrophilic killing by higher oxygen delivery and delaying or totally inhibiting the growth of fungal spores and mycelium (15).Rehabilitation or closure of the existing oronasal fistula can be done surgically or by construction of a prosthetic appliance.

Mucormycosis is a rapidly progressive disease with a fulminant course and fatal outcome unless diagnosed early and treated rapidly. Prognosis is dependent on multiple factors and early initiation of treatment is an important element. A multi-disciplinary approach consisting of dental specialists, ENT surgeons, ophthalmologists and neurologist is critical in successful management of a patient with mucormycosis.

\section{Acknowledgement}

I would like to thank our Principal and Prof Dr. H. N. Shama Rao and the department of Prosthodontics for their support and guidance.

\section{References}

1. Kyrmizakis DE, Doxas PG, Hajiioannou JK, Papadakis CE. Palate ulcer due to mucormycosis. J Laryngol Otol. 2002; 116:146-7.

2. Bouza E, Munoz P, Guinea J. Mucormycosis: an emerging disease? Clin Microbiol Infect. 2006; 12:7-23.

3. Kontoyiannis DP, Wessel VC, Bodey GP, Rolston KV. Zygomyco- sis in the 1990s in a tertiary-care cancer center. Clin Infect Dis. 2000; 30:851-6.

4. Succar MB, Nichols RD, Burch KH.Rhinocerebral mucormycosis. Arch Otolaryngol. 1979; 105:212-4.

5. Yohai RA, Bullock JD, Aziz AA, Markert RJ. Survival factors in rhino-orbital-cerebral mucormycosis. Surv Ophthalmol. 1994; 39:3-22.

6. Sugar AM. Mucormycosis. Clin Infect Dis. 1992; 14:S126-9.

7. Pak J, Tucci VT, Vincent AL, Sandin RL, Greene JN. Mucormycosis in immunochallenged patients. J Emerg Trauma Shock. 2008; 1:106-13.

8. Auluck A. Maxillary necrosis by mucormycosis. a case report and literature review. Med Oral Patol Oral Cir Bucal. 2007; 12:E360-4.

9. Salisbury PL, Caloss R Jr, Cruz JM, Powell BL, Cole R, Kohut RI. Mucormycosis of the mandible after dental extractions in a patient with acute myelogenous leukemia. Oral Surg Oral Med Oral Pathol Oral Radiol Endod. 1997; 83:340-4.

10. Tugsel Z, Sezer B, Akalin T. Facial swelling and palatal ulceration in a diabetic patient. Oral Surg Oral Med Oral Pathol Oral Radiol Endod. 2004; 98:630-6.

11. Van der Westhuijzen AJ, Grotepass FW, Wyma G, Padayachee A. A rapidly fatal palatal ulcer: rhinocerebral mucormycosis. Oral Surg Oral Med Oral Pathol. 1989; 68:32-6.

12. Ribes JA, Vanover-Sams CL, Baker DJ. Zygomycetes in human disease. Clin Microbiol Rev. 2000; 13:236-301.

13. Cagatay AA, Oncü SS, Calangu SS, Yildirmak TT, Ozsüt HH, Eraksoy HH. Rhinocerebral mucormycosis treated with 32 gram liposomal amphotericin B and incomplete surgery: a case report. BMC Infect Dis. 2001; 1:22.

14. Koçak R, Tetiker T, Koçak M, Baplamipli F, Zorludemir S, Gönlüpen $\mathrm{G}$. Fluconazole in the treatment of three cases of mucormycosis. Eur J Clin Microbiol Infect Dis. 1995; 14:559-61.

15. Couch L, Theilen F, Mader JT. Rhinocerebral mucormycosis with cerebral extension successfully treated with adjunctive hyperbaric oxygen therapy. Arch Otolaryngol Head Neck Surg. 1988; 114:791-4. 